\title{
Explicit solution for some generalized fluids in laminar flow with slip boundary conditions
}

\author{
Mourad Chamekha,b,*, Tarig. M. Elzaki ${ }^{a}$ \\ a Mathematics Department, Colleges of Science and Arts, AlKamel, University of Jeddah, KSA. \\ ${ }^{b}$ University of Tunis El Manar, National Engineering School at Tunis, LAMSIN, 1002, Tunis, Tunisia.
}

\begin{abstract}
In this study, we present a new approximation method to give an explicit solution of a laminar flow using a Sisko model. This is a problem of a generalized Newtonian fluid with slip boundary conditions. The proposed method is based on the variational iteration method (VIM) combined with an approximation step. This method is validated where the exact solution is available. In addition, in order to enrich the discussion, a numerical method is presented. The results illustrate that the VIM may be more effective that the finite difference method for a dilatant fluid. However, the VIM will be inappropriate for pseudoplastic fluid cases.
\end{abstract}

Keywords: Sisko model, variational iteration method, dilatant fluid, pseudoplastic fluid.

2010 MSC: 47E05, 35F30.

(C)2018 All rights reserved.

\begin{tabular}{|lc|}
\hline \multicolumn{2}{|l|}{ Nomenclature } \\
$u$ & velocity \\
$p$ & pressure \\
$\eta$ & viscosity \\
$\dot{\gamma}$ & rate shear \\
\hline
\end{tabular}

\section{Introduction}

The development of a new industrial fluid and the quality improvement of the existing products require knowledge of their material properties. The study of this fluid types takes into account many physical parameters that can essentially influence their behaviors. Examples of these effects, are magnetic, thermal radiation, nanoparticles and magnetohydrodynamics [3, 6, 8, 13]. Specifically, we must be familiar with the rheology of these products. Therefore, we will explore the behaviors of these fluids during a velocity field or under an applied pressure. To ensure a reasonable approach, we will propose to study a generalized Newtonian fluid. An example of these types is the non-Newtonian fluids that have a complex

\footnotetext{
${ }^{*}$ Corresponding author

Email address: chamekhmourad1@gmail.com \& mourad.chamekh@enit.utm.tn (Mourad Chamekh)

doi: $10.22436 /$ jmcs.018.03.03
}

Received: 2017-04-01 Revised: 2017-05-02 Accepted: 2017-05-29 
rheology $[2,4,7]$. These fluid types are a widespread phenomenon; it is of great relevance in big fields of physics. Amongst these, the fluids that run a pioneering part in the performance of beauty, gastronomy and care products. Here, we analyze the case of a Sisko model with slip boundary conditions in Couette and Poiseuille flows. According to [9], we show that in the laminar flow case the problem is as follows:

$$
\begin{gathered}
\frac{\partial u}{\partial y}+\alpha\left(\frac{\partial u}{\partial y}\right)^{m}-\beta y=0, \\
u(0)=b,
\end{gathered}
$$

with $u$ a velocity vector, for $\alpha=\frac{a}{\mu_{\infty}}, \beta=\frac{p_{x}}{\mu_{\infty}}, p_{x}$ is the gradient of the pressure. Usually, the boundary condition is chosen for $y=-h$ and $y \in[-h, h]$. For a simple reason, we have preferred (1.1b). The parameter $m$ is subject to fluid. It is called the flowing behavior index. If $m<1$, it is the pseudoplastic fluid. If $m>1$, it represents the dilatant fluid. If $m=1$, it may represent the Newtonian fluid. The parameters that characterize Sisko model are: The coefficient $a$ is the consistency coefficient and $\mu_{\infty}$, that is, the infinite shear rate viscosity [14].

$$
\eta(\dot{\gamma})=\mu_{\infty}+\mathrm{a}|\dot{\gamma}|^{m-1},
$$

where $\eta$ is the fluid viscosity, that is a function of rate shear $\dot{\gamma}$. We note that Sisko model plays a significant role in the modeling of fluids. If $m=1$ or $a=0$, we obtain Newtonian fluid. If $\mu_{\infty}=0$ the relation (1.2) recovers the generalized power law model. In addition, Weir and Bailey in [16], have been selected Sisko model from twenty compared models. The choice of Sisko model is justified by the fact which many research areas having been using it. Among those are, for example, the blood $[1,5]$ and the lubricating greases [14]. The solution of the problem could be acquired numerically. But the full analytic solution cannot be achieved unless for a few particular cases, $m=0.5, m=1$ (linear), $m=2$ and $m=3$ Ferrás et al. [9]. In this paper, we propose a new approximation method to resolve a problem of laminar flow of a generalized Newtonian fluid with slip boundary conditions using a Sisko model. This method is based on the variational iteration method (VIM) combined with an approximation step. The aim of the approximation is to relax the calculus of the integral. The efficiency of this method is shown in the cases where the exact solution is accessible.

\section{Problem solving}

Using the VIM, we can obtain an explicit approximate solution. But in this problem, unfortunately, we will show that most, if $m$ is not an integer number, the processes of calculus by VIM are confronted with a not obvious integration. To address this problem, we propose in the following subsections two stages to solving the problem (1.1).

\subsection{Variational iteration method step}

The analysis of many physical questions leads to a nonlinear differential problem. Sometimes, we could not have an exact solution. If we are able to do so, we have many difficult procedures of calculus to handle. We can overcome these problems with the variational iteration method. This is a method proposed by He [10] and developed by several authors. For more details, see, for example, Wazwaz [15] and Martin [11]. Besides, the VIM gives an efficient procedure to determine an analytic solution for some class of nonlinear problems. In addition, we note the simplicity of this method in taking into account the boundary conditions. Hence, we will show that this technique can have an analytical approximation of the solution by only a few simple steps of calculus. The general principle of the VIM is as follows. We consider the equation:

$$
\mathcal{L u}(x, y)+\mathcal{N u}(x, y)=g(x, y),
$$


with $\mathcal{L} u(x, y)=u_{y}$ design the linear part of equation, $\mathcal{N u}(x, y)=\alpha\left(u_{y}\right)^{m}$ is the nonlinear part and $g(x, y)=\beta y$ is the free term. In this conditions of problem (1.1), the gradient of velocity $\nabla u=u_{y}$. For this reason, we noted that $u(x, y)=u(y)$. The partial derivative $\frac{\partial}{\partial y}(\cdot)$ is denoted by $(\cdot)_{y}$.

The $n+1$-th approximate solution of (1.1) is generally given by

$$
u_{n+1}(y)=u_{n}(y)+\int_{0}^{y} \lambda(\xi)(\mathcal{L} \tilde{u}(\xi)+\mathcal{N} \tilde{u}(\xi)-g(\xi)) d \xi,
$$

with $\lambda(\xi)$ is a Lagrangian multiplier and $\tilde{u}(\xi)$ is considered as a restricted variation function, such $\delta \tilde{u}(\xi)=$ 0 . Then

$$
\delta u_{n+1}(y)=\delta u_{n}(y)+\int_{0}^{y} \lambda(\xi)\left(\delta u_{n y}(\xi)+\alpha \delta u_{n y}(\xi)^{m}-g(\xi)\right) d \xi
$$

Using integration by parts on (2.1), we show in this case that the Lagrangian multiplier $\lambda(\xi)=-1$.

To begin the calculus of the solution, we put

$$
u_{0}(y)=u(0)=b .
$$

The iteration formula becomes:

$$
u_{n+1}(y)=u_{n}(y)-\int_{0}^{y}\left(u_{n y}(\xi)+\left(u_{n y}(\xi)\right)^{m}-\beta \xi\right) d \xi .
$$

Then, for $n \geqslant 1$, we obtain the following reduced iteration formula

$$
u_{n+1}(y)=b+\frac{\beta}{2} y^{2}-\alpha \int_{0}^{y} u_{n y} \xi^{m} d \xi .
$$

The solution of the problem (1.1) is given by $u(y)=\lim _{n \rightarrow \infty} u_{n}(y)$. Therefore, $u_{0 y}(y)=0$ then

$$
\mathrm{u}_{1}(\mathrm{y})=\mathrm{b}+\frac{\beta}{2} \mathrm{y}^{2}
$$

The second order approximation of solution

$$
u_{2}(y)=b+\frac{\beta}{2} y^{2}-\frac{\alpha \beta^{m}}{m+1} y^{m+1} .
$$

Similarly, we obtain

$$
u_{3}(y)=b+\frac{\beta}{2} y^{2}-I_{m}(y)
$$

With

$$
I_{m}(y)=\alpha \int_{0}^{y}\left(\beta^{m} \xi^{m}\left(1-\alpha \beta^{m-1} \xi^{m-1}\right)\right)^{m} d \xi .
$$

We are going to propose the third order approximation of solution $\mathfrak{u}_{3}$ as an explicit approximate solution in the continuation of this paper.

\subsection{Approximation step}

By means of binomial formula, the exact integral $\mathrm{I}_{m}$ in (2.3) can easily be calculated (polynomial function), in which case $\mathrm{m}$ is an integer. Otherwise, we can evaluate this integral with a higher order approximation using the generalized binomial formula. We obtain the infinite sum:

$$
\left(1-\alpha \beta^{m-1} \xi^{m-1}\right)^{m}=\sum_{k=0}^{\infty}\left(\begin{array}{l}
k \\
m
\end{array}\right) \alpha^{k}(\beta y)^{k(m-1)} .
$$


Based on (2.4), we propose the following approximation:

$$
\begin{aligned}
\left(1-\alpha \beta^{m-1} y^{m-1}\right)^{m}= & 1-m \alpha \beta^{m-1} y^{m-1}+\frac{\alpha^{2} \beta^{2 m-2} m(m-1)}{2} y^{2 m-2} \\
& -\frac{\alpha^{3} \beta^{3 m-3} m(m-1)(m-2)}{6} y^{3 m-3} .
\end{aligned}
$$

As regards to the physical problem, frequently, we have $y \in[-h, h]$ and $x \in[0, L]$ with generally $h \ll L$. In addition to this, it is often reasonable to consider the analyzed equation in its a dimensionless form. Then, we can obtain a reasonable precision if we take $h$ as relatively lower to 1 .

By integrating the expression (2.5), we have

$$
\begin{aligned}
I_{m}(y)= & \frac{\alpha \beta^{m}}{m+1} y^{m+1}-\frac{\alpha^{2} \beta^{2 m-1}}{2} y^{2 m}+\frac{\alpha^{3} \beta^{3 m-2} m(m-1)}{2(3 m-1)} y^{3 m-1} \\
& -\frac{\alpha^{4} \beta^{4 m-3} m(m-1)(m-2)}{12(2 m-1)} y^{4 m-2}
\end{aligned}
$$

if $\mathrm{m} \neq \frac{1}{3}, \frac{1}{2}$.

\section{Numerical method}

We propose to find a numerical solution to problem (1.1) with a finite difference method. We have two main objectives:

First, to present a numerical method in the cases where the proposed method failed to give a precise solution. Second, to validate the results obtained in this study, particularly in the case where the expression of exact solution is not accessible. To do this, we begin to define a set of $N$ grid points $y_{j}$ in the domain $[0, h]$, we choose a step size $\Delta y=\frac{h}{N}$. The partial derivatives $\frac{\partial u}{\partial y}$ is approximated by central difference quotients. We obtain the following two steps numerical algorithm:

- Find $X_{k}$ solution of $X_{k}+\alpha\left(X_{k}\right)^{m}-\beta y_{k}=0$.

- Find the velocity $\mathfrak{u}_{\mathrm{k}+1}=\mathfrak{u}_{\mathrm{k}-1}+2 \Delta y X_{\mathrm{k}}$.

We note that first step consists of finding the approximation of partial derivative $X=\frac{\partial u}{\partial y}$, where we have used the Newton-Raphson to do so. The second consists in the approach of the fluid velocity $u$. We have written a Matlab code to implement this algorithm using the Matlab function "fsolve" for the Newton-Raphson method.

\section{Numerical examples}

As noted above, the parameter $m$ depending on the nature of the fluid. Following the values of $m$, we could distinguish:

Example 4.1 ( $m=2$ (Dilatant fluid)). We substitute $m=2$ in the expression (2.6), we obtain

$$
I_{2}(y)=\frac{\alpha \beta^{2}}{3} y^{3}-\frac{\alpha^{2} \beta^{3}}{2} y^{4}+\frac{\alpha^{3} \beta^{4}}{5} y^{5} .
$$

By using (2.2), the third approximated solution is given by

$$
u_{3}(x, y)=b+\frac{\beta}{2} y^{2}-\frac{\alpha \beta^{2}}{3} y^{3}+\frac{\alpha^{2} \beta^{3}}{2} y^{4}-\frac{\alpha^{3} \beta^{4}}{5} y^{5} .
$$


The exact solution can be calculated (see the Appendix). Otherwise, to obtain an objective comparison between the exact solution $u_{e x}$ and the proposed one $u_{3}$ with three parameters $\alpha, \beta$ and $b$, we have developed $u_{e x}$ in Taylor series

$$
u_{e x}(x, y)=b+\frac{\beta}{2} y^{2}-\frac{\alpha \beta^{2}}{3} y^{3}+\frac{\alpha^{2} \beta^{3}}{2} y^{4}-\alpha^{3} \beta^{4} y^{5}+\frac{7 \alpha^{4} \beta^{5}}{3} y^{6}+\cdots
$$

Then we obtain

$$
\left|u_{e x}(y)-u_{3}(y)\right|=\frac{4 \alpha^{3} \beta^{4}}{5}|y|^{5} \leqslant C_{\alpha \beta} h^{5} .
$$

Theoretically, the VIM solution converges to the exact solution. In this study, we have combined an analytic computing process with an approximate formula (2.4), in order to prevent the complicated integral problems.

Table 1: The point-to-point comparison between the errors of VIM and the numerical method for $m=2$.

\begin{tabular}{lll}
\hline$y$ & VIM & Numerical method \\
\hline 0 & 0 & 0 \\
0.1 & $4 \times 10^{-7}$ & $7 \times 10^{-5}$ \\
0.2 & $1 \times 10^{-5}$ & $5 \times 10^{-4}$ \\
0.3 & $8 \times 10^{-5}$ & $1.8 \times 10^{-3}$ \\
0.4 & 0.0003 & 0.0041 \\
0.5 & 0.0008 & 0.0077 \\
0.6 & 0.0019 & 0.0128 \\
0.7 & 0.0038 & 0.0194 \\
0.8 & 0.0067 & 0.0279 \\
0.9 & 0.0111 & 0.0382 \\
1.0 & 0.0173 & 0.0506 \\
\hline
\end{tabular}

Example 4.2 ( $m=1.5$ (Dilatant fluid and non-integer index)). If $m=\frac{3}{2}$, using (2.2) and (2.6), we obtain

$$
\mathrm{I}_{1.5}(\mathrm{y})=\frac{\beta^{1.5}}{2.5} \mathrm{y}^{2.5}-\frac{\alpha \beta^{2}}{2} \mathrm{y}^{3}+\frac{3 \alpha^{2} \beta^{2.5}}{28} \mathrm{y}^{3.5}+\frac{3 \alpha^{3} \beta^{3}}{96} \mathrm{y}^{4}+\frac{9 \alpha^{4} \beta^{3.5}}{2640} \mathrm{y}^{4.5}
$$

Thus,

$$
u_{3}(x, y)=b+\frac{\beta}{2} y^{2}+\frac{\beta^{1.5}}{2.5} y^{2.5}-\frac{\alpha \beta^{2}}{2} y^{3}+\frac{3 \alpha^{2} \beta^{2.5}}{28} y^{3.5}+\frac{3 \alpha^{3} \beta^{3}}{96} y^{4}+\frac{9 \alpha^{4} \beta^{3.5}}{2640} y^{4.5}
$$

Example 4.3 ( $m=0.5$ (Pseudoplastic fluid case)). If $m=\frac{1}{2}$ and using (2.2) and (2.6) we obtain

$$
\mathrm{I}_{0.5}(\mathrm{y})=\frac{\alpha \beta^{0} .5}{1.5} y^{1.5}-\frac{\alpha^{2}}{2} y-\frac{\alpha^{3}}{4} \beta^{-0.5} y^{0.5}
$$

Thus

$$
u_{3}(y)=b+\frac{\beta}{2} y^{2}+\frac{\alpha \beta^{0} .5}{1.5} y^{1.5}-\frac{\alpha^{2}}{2} y-\frac{\alpha^{3}}{4} \beta^{-0.5} y^{0.5}
$$

As in the case for $m=2$, we could obtain the full analytical solution developed in Appendix for $m=\frac{1}{2}$ :

$$
u_{e x}(y)=\frac{1}{12} \beta\left(\alpha^{2}+4 \beta y\right)^{1.5}-\frac{\alpha}{2} y+b-\frac{\alpha^{3}}{12} \beta .
$$


The development of $u_{\mathrm{ex}}$ in Taylor series is given by

$$
u_{e x}(y) \approx b-\frac{\alpha^{3} \beta}{12}+\frac{\alpha^{3}}{12 \beta}+\frac{\beta y^{2}}{2 \alpha}-\frac{\beta^{2} y^{3}}{3 \alpha^{3}}+\frac{\beta^{3} y^{4}}{2 \alpha^{5}}
$$

To compare the VIM solution and the exact solution, with neglecting the higher-order terms, thus we obtain the following expression:

$$
\left(u_{e x}(y)-u_{3}(y)\right)=-\frac{\alpha^{3} \beta}{12}+\frac{\alpha^{3}}{12 \beta}+\frac{\alpha^{3}}{4} \beta^{-0.5} y^{0.5}+\frac{\alpha^{2}}{2} y-\frac{\alpha \beta^{0} .5}{1.5} y^{1.5}+\left(\frac{\beta}{2 \alpha}-\frac{\beta}{2}\right) y^{2} .
$$

The expression (4.2) shows that the difference between the exact solution and the VIM one leads to a not negligible quantity, we conclude that result is imprecise for $m=\frac{1}{2}$.

\subsection{Comparison of the results}

In order to test the efficiency of the VIM, we have proposed some numerical essays. We discuss the results obtained by applying both methods. For those purposes, the selected physical parameters appear in dimensionless form. We chose $h=1$, the step size $\Delta y=0.05, b=1, \alpha=1,0.1$ and $\beta=0.5,0.05$.

As the results in Figure 1, the calculation of the velocity by the numerical method and VIM is in good agreement with the exact solution with a behavior index $m=2$ and parameters $\alpha=0.1$ and $\beta=0.05$. In turn, the VIM proves more efficiency compared to the proposed numerical method if $\alpha=1$ and $\beta=0.5$ (see Figure 2 and Table 1).

In Figure 3 the numerical method starts off approximating the VIM solution reasonably well. With the behavior index $m=\frac{3}{2}$ and parameters $\alpha=1$ and $\beta=0.05$. That is a sensitive case as the exact solution is not known.

Figure 4 shows the harmony between the numerical and exact solution. That is a case when the VIM is failed to give a reasonable result. The behavior index is chosen $m=\frac{1}{2}$ and parameters $\alpha=1$ and $\beta=0.05$.

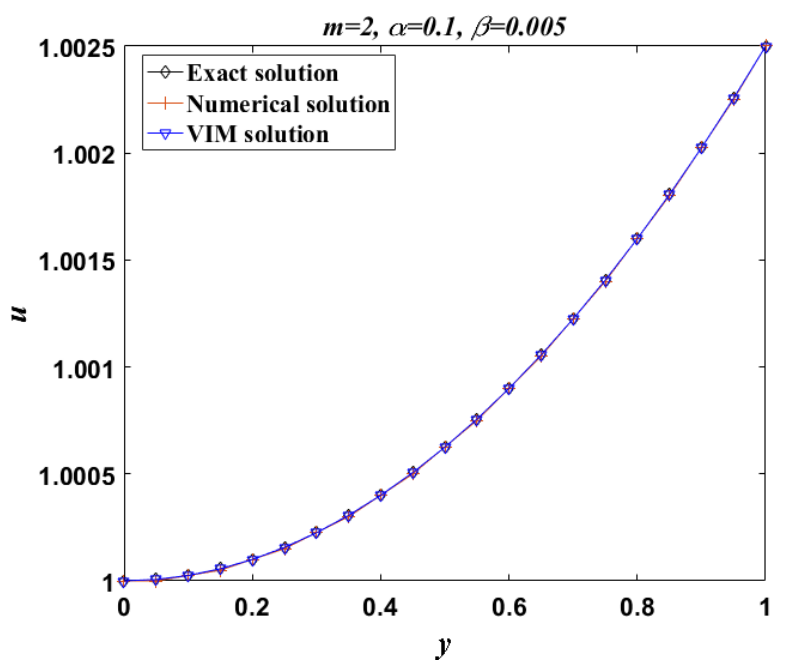

Figure 1: Precise results are shown in this figure using the VIM and the numerical method. Noting that the three values of the velocity are confused. 


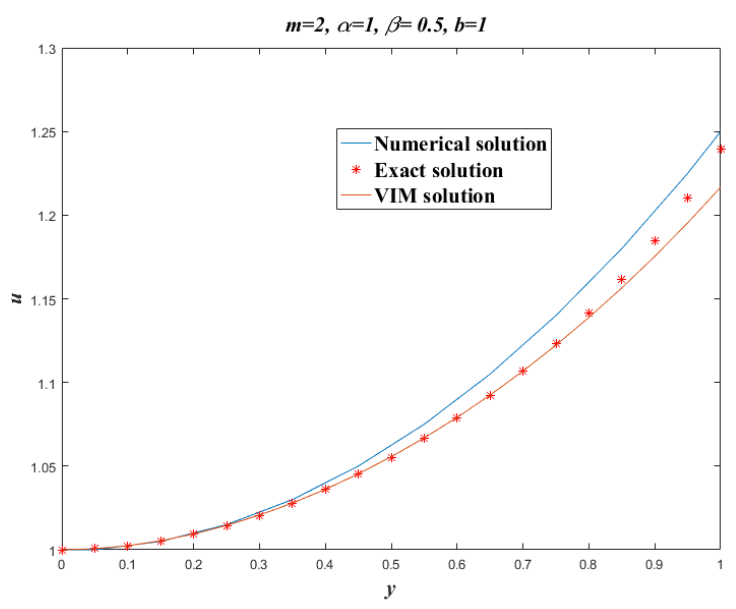

Figure 2: This figure shows that VIM is more precise compared the proposed numerical method.



Figure 3: Curves of velocity calculated by VIM in blue line (solid line) and numerical method (red or dashed line). (Dilatant fluid and non-integer index $\mathrm{m}=1.5)$.

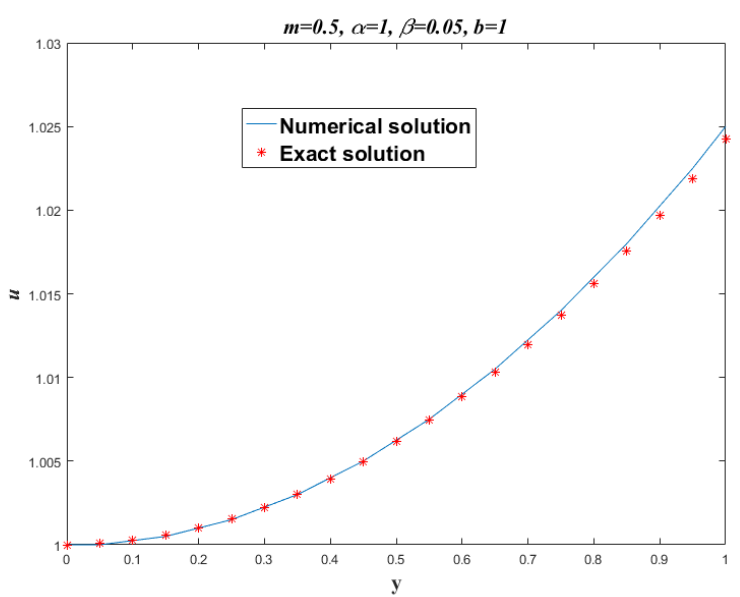

Figure 4: Curve of velocity calculated by the numerical method in blue solid line compared to exact velocity in red stars (Pseudoelastic fluid with index $\mathrm{m}=0.5$ ). 


\subsection{Discussions}

Discussions: To solve the problem (1.1), we note that the VIM is an effective method. By using the approximation (2.4), we have overcome the integral difficulty. For a dilatant fluid $(\mathrm{m}>1)$, the approximate solution seems to show the best precision often in the case $m=2$, when the calculated solution is compared to the exact solution. Should this not be the case, we have to use the numerical technique to confirm the efficiency of the VIM solution. However, in the pseudoplastic $(m<1)$ case, the proposed VIM method does not reach the same result. As one can see in the expression (4.2), the term on the right obviously not negligible. In Addition, It is clear that in the expression of the VIM solution (4.1) the exponent of y decrease. So, if we go further into the calculus to attain more precision, it leads to the appearance of singular terms with a nonpositive exponent. That can affect the convergence quality. We conclude in this case of pseudofluid, that this method is obviously not efficient. It means that we have to apply the numerical method presented in the Section 3 in order to find the solution for this fluid type.

\section{Conclusion}

An approximation method for laminar flow of a generalized Newtonian fluid with slip boundary conditions using a Sisko model has been presented in this paper. Exceptionally, for few particular values of $m$, the analytic solution of the equation (1.1) is explored. Generally, it is not easy to calculate the solution, such as the existence of the nonlinear term related to this exponent $\mathrm{m}$. Through a novel approach based on the VIM and combined with an approximate step, we have obtained an explicit approximated solution with rapid convergence in the dilatant fluid case, without using restrictive assumptions. This method has not proved effective in pseudofluid case. In order to enrich this analysis, we have proposed to find a numerical solution to the problem (1.1) with the finite difference method. We have targeted two objectives: first, to present a numerical method in the cases where the proposed method is failing to give a precise solution; and second, to validate the results obtained in this paper. We have concluded that: For a dilatant fluid, the numerical results show that the VIM had the best numerical performance both in accuracy and in speed, especially compared to the proposed numerical method. However, the VIM fails to get a result for a pseudoplastic fluid. For this latter, the numerical method is the most appropriate method to use. In future attempts, we recommend to find the analytical solutions to the pseudofluid case and extend the acquired result of other fluid types with more complex forms.

\section{Appendix A: Analytical solutions}

We will discuss in this appendix the existence of an analytic solution for the problem (1.1). Following the method used by Polyanin and Zaitsev in [12], we can propose an exact solution for any behavior index $\mathrm{m}$, but these solutions are given only in parametric form. We believe that the full analytic form is only possible for specific cases we will treat separately.

We consider the (1.1a)

The general form is given by

$$
u_{y}+\alpha\left(u_{y}\right)^{m}-\beta y=0 .
$$

$$
y=f\left(u_{y}\right)=\frac{1}{\beta} u_{y}+\frac{\alpha}{\beta}\left(u_{y}\right)^{m} .
$$

Putting $t=u_{y}$, we obtain

$$
\begin{aligned}
y & =f(t)=\frac{1}{\beta} t+\frac{\alpha}{\beta} t^{m} \\
\frac{d u}{d t} & =\frac{d u}{d y} \frac{d y}{d t}=t f^{\prime}(t)=\frac{1}{\beta} t+\frac{m \alpha}{\beta} t^{m} .
\end{aligned}
$$

Thus

$$
u=\int t f^{\prime}(t) d t=\frac{1}{2 \beta} t^{2}+\frac{m \alpha}{\beta(m+1)} t^{m+1}+C .
$$


One can obtain

$$
\begin{aligned}
& y=\frac{1}{\beta} t+\frac{\alpha}{\beta} t^{m}, \\
& u=\frac{1}{2 \beta} t^{2}+\frac{m \alpha}{\beta(m+1)} t^{m+1}+C .
\end{aligned}
$$

The treatable cases are $m \in\{0,0.5,1,2,3\}$ because one can easily give an explicit expression of $t$ to respect $y\left(t=f^{-1}(y)\right)$ using (5.1a). Submitting that expression of $t$ in (5.1b), we can obtain an expression analytic of the velocity $u$. We note that the cases $m=0$ and $m=1$ are obvious.

Case $m=2$ : Solving the equation $\alpha t^{2}+t-\beta y=0$, we obtain $t=\frac{-1 \pm \sqrt{1+4 \alpha \beta y}}{2 \alpha}$. For a physical admissibility reason $\left(\frac{d u}{d t}>0\right)$ and adding the boundary condition $(1.1 b)$, we can propose the following solution:

$$
u_{e x}(y)=\frac{1}{12 \alpha^{2} \beta}(1+4 \alpha \beta y)^{\frac{3}{2}}-\frac{1}{2 \alpha} y+b-\frac{1}{12 \alpha^{2} \beta} .
$$

Case $m=0.5$ : This case is similar to last. But we solve $Z^{2}+\alpha Z-\beta y=0$, with $Z^{2}=t$. Then

$$
u_{e x}(y)=\frac{1}{12 \beta}\left(\alpha^{2}+4 \beta y\right)^{\frac{3}{2}}-\frac{\alpha}{2} y+b-\frac{\alpha^{3}}{12 \beta} .
$$

Case $m=3$ : The solution of equation $\alpha t^{3}+t-\beta y=0$, is given by it classical form

$$
t=\left(q+\left[q^{2}+r^{3}\right]^{1 / 2}\right)^{1 / 3}+\left(q-\left[q^{2}+r^{3}\right]^{1 / 2}\right)^{1 / 3}
$$

where $\mathrm{q}=\frac{\beta y}{2 \alpha}$ and $\mathrm{r}=\frac{1}{3 \alpha}$. Respecting the physical admissibility of the problem we can propose an analytic solution. For simplicity we treat the problem with $\beta=2 / 3$ and $\alpha=1 / 3$. Then $q=y, r=1$ and

$$
t=\left(y+\left[y^{2}+1\right]^{1 / 2}\right)^{1 / 3}+\left(y-\left[y^{2}+1\right]^{1 / 2}\right)^{1 / 3} .
$$

Thus

$$
u(y)=\frac{1}{3}\left\{\left(y+\left[y^{2}+1\right]^{1 / 2}\right)^{1 / 3}+\left(y-\left[y^{2}+1\right]^{1 / 2}\right)^{1 / 3}\right\}^{2}+\frac{3}{8}\left\{\left(y+\left[y^{2}+1\right]^{1 / 2}\right)^{1 / 3}+\left(y-\left[y^{2}+1\right]^{1 / 2}\right)^{1 / 3}\right\}^{4}+b .
$$

\section{Acknowledgment}

The authors are grateful to the anonymous referees valuable comments that improved the manuscript.

\section{References}

[1] N. Ali, A. Zaman, M. Sajid, Unsteady blood flow through a tapered stenotic artery using Sisko model, Comput. \& Fluids, 101 (2014), 42-49. 1

[2] M. M. Bhatti, T. Abbas, M. M. Rashidi, Numerical Study of Entropy Generation with Nonlinear Thermal Radiation on Magnetohydrodynamics non-Newtonian Nanofluid Through a Porous Shrinking Sheet, J. Magnet., 21 (2016), 468-475. 1

[3] M. M. Bhatti, R. Ellahi, A. Zeeshan, Study of variable magnetic field on the peristaltic flow of Jeffrey fluid in a non-uniform rectangular duct having compliant walls, J. Mol. Liq., 222 (2016), 101-108. 1

[4] M. M. Bhatti, A. Zeeshan, Analytic study of heat transfer with variable viscosity on solid particle motion in dusty Jeffery fluid, Modern Phys. Lett. B, 2016 (2016), 13 pages. 1

[5] M. M. Bhatti, A. Zeeshan, R. Ellahi, Endoscope analysis on peristaltic blood flow of Sisko fluid with Titanium magnetonanoparticles, Comput. Biol. Med., 78 (2016), 29-41. 1 
[6] M. M. Bhatti, A. Zeeshan, N. Ijaz, O. A. Beg, A. Kadir, Mathematical modelling of nonlinear thermal radiation effects on EMHD peristaltic pumping of viscoelastic dusty fluid through a porous medium duct, Eng. Sci. Tech. Int. J., 20 (2017), 1129-1139. 1

[7] R. Ellahi, M. M. Bhatti, I. Pop, Effects of Hall and Ion Slip on MHD peristaltic flow of Jeffrey fluid in a non-Uniform rectangular duct, Internat. J. Numer. Methods Heat Fluid Flow, 26 (2016), 1802-1820. 1

[8] R. Ellahi, E. Shivanian, S. Abbasbandy, T. Hayat, Numerical study of magnetohydrodynamics generalized Couette flow of Eyring-Powell fluid with heat transfer and slip condition, Internat. J. Numer. Methods Heat Fluid Flow, 26 (2016), 1433-1445. 1

[9] L. L. Ferrás, J. M. Nóbrega, F. T. Pinho, Analytical solutions for Newtonian and inelastic non-Newtonian flows with wall slip, J. Non-Newtonian Fluid Mech., 175-176 (2012), 76-88. 1, 1

[10] J.-H. He, Variational iteration method a kind of non-linear analytical technique: some examples, Int. J. Nonlinear Mech., 34 (1999), 699-708. 2.1

[11] O. Martin, Modified variational iteration method for the analysis of viscoelastic beams, Appl. Math. Model., 40 (2016), 7988-7995. 2.1

[12] A. D. Polyanin, V. F. Zaitsev, Handbook of Exact Solutions for Ordinary Differential Equations, Second edition, Chapman \& Hall/CRC, Boca Raton, London, New York, (2003). 5

[13] S. U. Rahman, R. Ellahi, S. Nadeem, Q. M. Zaigham Zia, Simultaneous effects of nanoparticles and slip on Jeffrey fluid through tapered artery with mild stenosis, J. Mol. Liq., 218 (2016), 484-493. 1

[14] A. W. Sisko, The flow of lubricating greases, Ind. Eng. Chem., 50 (1958), 1789-1792. 1, 1

[15] A.-M. Wazwaz, The variational iteration method for solving a system of PDES, Comput. Math. Appl., 54 (2007), 895902. 2.1

[16] I. S. Weir, W. J. Bailey, A statistical study of rheological models for drilling fluids: Society of Petroleum Engineers, SPE Journal, 1 (1996), 473-486. 1 